\title{
Free-Piston Diesel Engine Dynamics and Control
}

\author{
Tor A. Johansen*1, Olav Egeland*, Erling Aa. Johannessen** 2 and Rolf Kvamsdal** \\ * Dept. Engr. Cybernetics, Norwegian Univ. Sci. Tech., N-7491 Trondheim, Norway. \\ ** Kværner ASA, Postboks 169, N-1325 Lysaker, Norway.
}

\begin{abstract}
Free-piston diesel engines are characterized by freely moving pistons without any rigid crankshaft or camshaft connected to the pistons. This allows a highly compact and efficient engine design, but requires automatic control of the piston motion. This paper present a dynamic mathematical model of a free-piston diesel engine, a control oriented dynamic analysis, and a novel computer-based piston motion control system. Experimental evaluation of the results on a full scale diesel test cylinder show feasibility of the suggested control approach.
\end{abstract}

\section{Introduction}

The free-piston diesel engine concept was developed by Pescara, and engines of various size were manufactured between 1930-1960 by GM, Ford, Renault, Junker, SIGMA and others [1]. Despite the potential advantages of the Pescara process, the mechanical construction and control mechanisms had weaknesses leading to low reliability. Partload operation of the engine was a known difficulty and the partload thermal efficiency was generally poor. Furthermore, lack of suitable materials imposed limitations on the maximum temperatures and thermal efficiency. Today, new materials, modern computer control technology, and high-precision common-rail diesel injection systems allows the implementation of an accurate and reliable electronic control system. This was the motivation for Kværner ASA, who is studying a modern high-speed free-piston diesel engine concept aimed at marine applications, as an alternative to both gas turbines and traditional diesel engines. The net output of an 8 cylinder engine is about $8 \mathrm{MW}$ with a thermal efficiency of about $50 \%$. In contrast to the original free piston diesel engine with an opposed piston arrangement with two opposed pistons moving syncrhonously in the same cylinder, the Kværner design is a more conventional two-stroke arrangement with with a single piston per cylinder and a standard diesel cylinder top with a centered injector and four poppet exhaust values per cylinder.

With the exception of the recent work [2], which is based on a different type of free-piston engine concept, there are no other results on electronic control of freepiston diesel engines reported in the literature. The contribution of the present paper is that it present dynamic models, energy-based control design, and experimental results on a full scale test cylinder. In [3] we give details of the engine control system, emphasizing valve

\footnotetext{
${ }^{1}$ Corresponding author: Tor.Arne.Johansen@itk.ntnu.no

${ }^{2}$ Present address: Rolls-Royce Marine AS, Postboks 924, 5808 Bergen, Norway
}

and injector timing, piston motion parameter estimation, signal processing, control and its implementation. The main ideas are described in the patent [4], also for an opposed piston arrangement.

\section{Free-piston diesel engine operating principles}

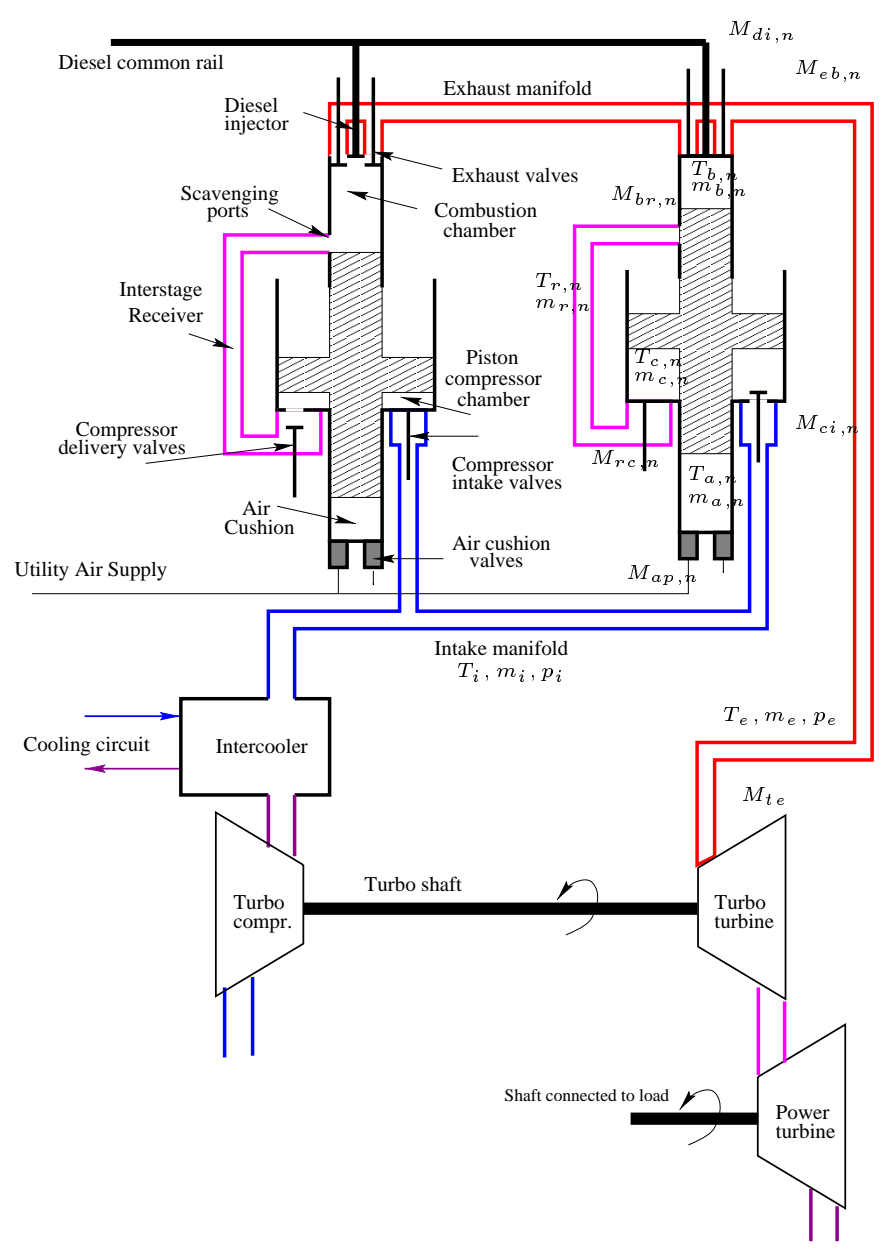

Figure 1: Sketch of multi-cylinder free-piston engine, indicating mass and energy flows.

Figure 1 provides a principal sketch of a multi-cylinder free-piston diesel engine. For cylinder/piston number $n$, the air cushion chamber is indicated by subscripts $a, n$, the combustion chamber by $b, n$, and the compression chamber by $c, n$. There is an interstage receiver chamber, denoted $r, n$, and common intake and exhaust manifolds denoted $i$ and $e$, respectively. The 
index $p$ refers to a utility air reservoir, and the ambient stagnation state is denoted 0 .

The two-stroke diesel cycle, seen from the intake manifold to the exhaust manifold, can be described as follows (see also [5]). Compression: The air in the combustion chamber is compressed when the piston moves from its bottom dead center towards its top dead center. Combustion: Near the top dead center, the temperature of the compressed air is high enough for autoignition, and diesel is injected at high pressure into the combustion chamber through a nozzle, and combustion of the diesel fuel results. Expansion: The high pressure in the combustion chamber makes the piston move downwards and the exhaust gas expands. Scavenging: During this downwards motion, the combustion finishes and the actively controlled exhaust valves opens at high pressure to deliver high-energy exhaust gas to the exhaust manifold and turbines. When the piston is at some distance from its nominal bottom dead center, scavenging ports are uncovered and the fresh air in the interstage receiver flushes the combustion chamber and replaces the exhaust gas. Compression in piston compressor: The interstage receiver is at the same time filled by high-pressure air that is compressed in the piston compressor chamber during the downwards motion of the piston and delivered through some actively controlled valves connecting the piston compressor chamber and interstage receiver. The high pressure in the compressor chamber and air cushion makes the piston move upwards from its bottom dead center. Air intake: During the upwards motion of the piston, fresh air is sucked into the compressor chamber from the intake manifold through passive suction valves.

The free-piston diesel engine differs from a traditional diesel engine in several ways: i) The pistons move freely, only influenced by pressure and friction forces since there is no crankshaft connected to the piston. ii) The exhaust and compressor delivery valves are controlled electronically, since there is no camshaft connecting them to the pistons. iii) The exhaust gas has much higher temperature and pressure than in a traditional engine, as the mechanical power is produced directly from the exhaust gas in the power turbine. The free-piston concept used in the present study is feasible only if the piston motion can be controlled. The requirements in terms on control accuracy and robustness are high as cycle-to-cycle variations in the motion of the piston at stationary running will introduce undesired vibrations and disturbances in the intake and exhaust manifold that will influence the operation, cost and life-cycle of the turbomachinery. The cycle-tocycle variability in stroke length should be less than $2 \mathrm{~mm}$ out of a stroke of about $200 \mathrm{~mm}$. Furthermore, there are hard constraints on the motion of the piston due to mechanical stop if the pistons hits the top or bottom of the cylinder. The absolute tolerance here is typically less than $8 \mathrm{~mm}$, depending on the operating point of the engine.

The motion of the piston can only be influenced through the mechanical work made by the gas pressures on the piston. These pressures can be indirectly influenced by controlling the mass flow through the engine by actively controlling the point when the hydraulically actuated exhaust and compressor delivery valves open and close. Furthermore, the pressures can be influenced by the amount of diesel being injected into the combustion chamber and by taking air in or out of the air cushion. In fact, the main purpose of the air cushion is to provide a mechanism for control, i.e. balancing the pressure forces on the piston.

\section{Mathematical Model of $N$-Cylinder Free-Piston Engine}

In this section we derive a mathematical model based on ordinary differential equations describing mass- and energy-balances of the main gas volumes in addition to a force balance for the pistons. The basic equations are derived under the assumption of the gas being ideal and that heat loss and friction are negligible. A somewhat more detailed model with similar structure but based on somewhat more realistic assumptions, including a simple model of the combustion chemistry, has been developed and used for simulation.

Dot notation is used to denote time differentiation so that $\dot{m}$ is the time derivative of $m$, that is, $\dot{m}=\frac{d}{d t} m$, and not the mass flow as in common in the combustion engine literature. Mass flow from $i$ to $j$ is denoted by $M_{j i}$. The variable $x$ is the piston position, $T$ denotes absolute temperature, $p$ denotes pressure, $V$ is volume, $\rho$ is density and $m$ is mass. The indices follows the notation introduced in Figure 1.

\subsection{Geometric parameters}

The inner piston diameter corresponding to the air cushion and combustion chamber is $d_{p i}$. The outer part of the piston corresponding to the compressor chamber has diameter $d_{p o}$. The mass of each of the pistons is $m$. The cross section areas are for the air cushion, combustion chamber and compression chambers:

$$
A_{a}=\frac{\pi}{4} d_{p i}^{2}, \quad A_{b}=\frac{\pi}{4} d_{p i}^{2}, \quad A_{c}=\frac{\pi}{4}\left(d_{p o}^{2}-d_{p i}^{2}\right)
$$

The volumes of the air cushion chambers are $V_{a, n}=$ $A_{a}\left(x_{0 a}-x_{n}\right)$, where $x_{0 a}$ is the effective length of each of the air cushions. The volumes of the combustion chambers are $V_{b, n}=A_{b}\left(x_{0 b}+x_{n}\right)$ where $x_{0 b}$ accounts for dead volume at the piston upper stop position, corresponding to mechanical contact with the cylinder top. The volumes of the compression chambers are $V_{c, n}=A_{c}\left(x_{0 c}-x_{n}\right)$ where $x_{0 c}$ is the effective length of the compression chamber, including dead volume at the piston lower stop position. The volume of the interstage receiver chamber and exhaust manifolds are $V_{r}$ and $V_{e}$ respectively.

The position $x_{n}$ increases when the piston moves downwards, cf. Figure 2. The piston is said to be at the top dead center (TDC) when $x_{n}$ is at its minimum for the actual cycle. The velocity at this point satisfies $\dot{x}_{n}=0$. The position at TDC for the actual cycle is defined to be $x_{n}^{T D C}$. Likewise, the bottom dead center (BDC) is denoted $x_{n}^{B D C}$. This position is reached when $x_{n}$ is at its maximum for the actual cycle. Some further geometric parameters are defined in Figure 2.

\subsection{Force balances}

Equations of motion for pistons:

$$
m \ddot{x}_{n}=-p_{a, n} A_{a}-p_{c, n} A_{c}+p_{0} A_{c}+p_{b, n} A_{b}
$$




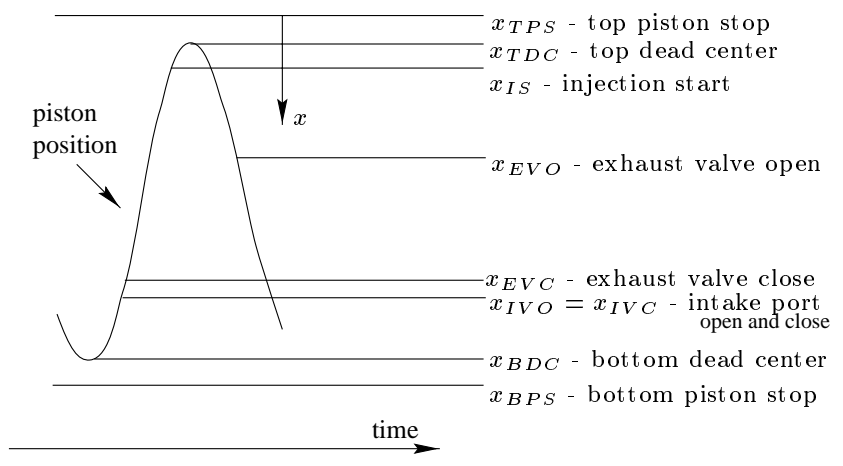

Figure 2: Geometric cycle parameters.

\subsection{Mass balances}

Conservation of mass gives

$$
\begin{aligned}
\dot{m}_{a, n} & =M_{a p, n} \\
\dot{m}_{b, n} & =M_{b r, n}-M_{e b, n} \\
\dot{m}_{c, n} & =M_{c i, n}-M_{r c, n} \\
\dot{m}_{r, n} & =M_{r c, n}-M_{b r, n} \\
\dot{m}_{e} & =\sum_{n=1}^{N} M_{e b, n}-M_{t e}
\end{aligned}
$$

The mass flows are expressed as $M_{a p, n}=M_{a p, n}^{\text {in }}-M_{a 0, n}^{\text {out }}$ where superscript "in" refers to flow in to the air cushion, while superscript "out" refers to flow out. The variables $M_{a p, n}^{\mathrm{in}}$ and $M_{a 0, n}^{\text {out }}$ are both non-negative. Likewise, $M_{b r, n}=M_{b r, n}^{\text {in }}-M_{b r, n}^{\text {out }}$ and $M_{e b, n}=M_{e b, n}^{\text {out }}-M_{e b, n}^{\text {in }}$ where $M_{b r, n}^{\mathrm{in}}, M_{b r, n}^{\text {out }}, M_{e b, n}^{\text {out }}$ and $M_{e b, n}^{\mathrm{in}}$ are nonnegative, and the superscript "in" refers to mass flow into the combustion chamber, while the superscript "out" refers to mass flow out of the combustion chamber. Finally, $M_{r c, n}=M_{r c, n}^{\text {out }}-M_{r c, n}^{\text {in }}$ where $M_{r c, n}^{\text {out }}$ and $M_{r c, n}^{\text {in }}$ are nonnegative. The superscript "in" refers to mass flow into the compressor chamber, while the superscript "out" refers to mass flow out of the compressor chamber.

\subsection{Energy balances}

The energy balances for the air cushion, compressor, combustion, interstage receiver and exhaust manifold chambers are for $n=1,2, \ldots, N$

$$
\begin{aligned}
& \dot{T}_{b, n}=\frac{M_{b r, n}^{\text {in }}}{m_{b, n}} \kappa T_{r, n}+\frac{M_{e b, n}^{\text {in }}}{m_{b, n}} \kappa T_{e} \\
& -\frac{M_{b r, n}^{\mathrm{in}}+M_{e b, n}^{\mathrm{in}}+\left(M_{b r, n}^{\text {out }}+M_{e b, n}^{\text {out }}\right)(\kappa-1)}{m_{b, n}} T_{b, n} \\
& -\frac{1}{m_{b, n} c_{v}} A_{b} p_{b, n} \dot{x}_{n}+\frac{h_{d i} M_{d i, n}}{m_{b, n} c_{v}} \\
& \dot{T}_{a, n}=\frac{M_{a p, n}^{\mathrm{in}}}{m_{a, n}}\left(\kappa T_{p}-T_{a, n}\right)-\frac{M_{a p, n}^{\mathrm{out}}(\kappa-1)}{m_{a, n}} T_{a, n} \\
& +\frac{1}{m_{a, n} c_{v}} A_{a} p_{a, n} \dot{x}_{n} \\
& \dot{T}_{c, n}=\frac{M_{c i, n}}{m_{c, n}}\left(\kappa T_{i}-T_{c, n}\right)-\frac{M_{r c, n}^{\mathrm{in}}+M_{r c, n}^{\mathrm{out}}(\kappa-1)}{m_{c, n}} T_{c, n} \\
& +\frac{M_{r c, n}^{\mathrm{in}}}{m_{c, n}} \kappa T_{r, n}+\frac{1}{m_{c, n} c_{v}} A_{c}\left(p_{c, n}-p_{0}\right) \dot{x}_{n}
\end{aligned}
$$

$$
\begin{aligned}
\dot{T}_{r, n}= & \frac{M_{r c, n}^{\text {out }}}{m_{r}} \kappa T_{c, n}+\frac{M_{b r, n}^{\text {out }}}{m_{r}} \kappa T_{b, n} \\
& -\frac{M_{r c, n}^{\text {out }}+M_{b r, n}^{\text {out }}+\left(M_{r c, n}^{\text {in }}+M_{b r, n}^{\text {in }}\right)(\kappa-1)}{m_{r}} T_{r, n} \\
\dot{T}_{e}= & \sum_{n=1}^{N}\left(\frac{M_{e b, n}^{\text {out }}}{m_{e}}\left(\kappa T_{b, n}-T_{e}\right)-\frac{M_{e b, n}^{\text {in }}}{m_{e}}(\kappa-1) T_{e}\right) \\
& -\frac{M_{t e}}{m_{e}}(\kappa-1) T_{e}
\end{aligned}
$$

where $\kappa=c_{p} / c_{v}$. The ambient density, density of the utility pressurized air, and density of the air in the intake manifold are

$$
\rho_{0}=\frac{p_{0}}{R T_{0}}, \quad \rho_{p}=\frac{p_{p}}{R T_{p}}, \quad \rho_{i}=\frac{p_{i}}{R T_{i}}
$$

where $R$ is the universal gas constant. The diesel heat release $M_{d i} h_{d i}$ is initiated at some piston position $x_{C S}$, and assumed to evolve according to some time-varying function that depends on the diesel pressure and injected amount, see e.g. $[5,6]$.

\subsection{Mass flows}

Mass flows through valves and ports are modeled as isentropic flow through nozzles. Let the inlet stagnation pressure be denoted $p_{1}$ and the nozzle exit pressure, which is assumed to be equal to the throat pressure, be denoted by $p_{2}$. Then the critical exit pressure corresponding to choked flow is

$$
p_{C}=\left(\frac{2}{\kappa+1}\right)^{\frac{\kappa}{\kappa-1}} p_{1}
$$

The mass flow through the valve is [5]

$$
M= \begin{cases}A \sqrt{\frac{2 \kappa}{\kappa-1} p_{1} \rho_{1}\left[\left(\frac{p_{2}}{p_{1}}\right)^{\frac{2}{\kappa}}-\left(\frac{p_{2}}{p_{1}}\right)^{\frac{\kappa+1}{\kappa}}\right]} & \text { if } p_{2}>p_{C} \\ A \sqrt{\kappa p_{1} \rho_{1}\left(\frac{2}{\kappa+1}\right)^{\frac{\kappa+1}{\kappa-1}}} & \text { if } p_{2} \leq p_{C}\end{cases}
$$

\subsection{Numerical values}

The parameters in Table 1 corresponds to the Kværner KLC test cylinder with about $1 \mathrm{MW}$ net output. At full load the thermal efficiency is about $50 \%$, mass flow through the engine is about $1.75 \mathrm{~kg} / \mathrm{s}$ per cylinder, stroke length about $189 \mathrm{~mm}$, intake pressure (from turbo compressor) about 3.25 bar, exhaust pressure about 20 bar, exhaust temperature about $1050 \mathrm{~K}$, and piston frequency about $1800 \mathrm{rpm}$.

\begin{tabular}{||l|l||l|l||}
\hline \hline$d_{p i}$ & $0.180 \mathrm{~m}$ & $d_{p o}$ & $0.430 \mathrm{~m}$ \\
\hline$x_{0 a}$ & $0.2945 \mathrm{~m}$ & $x_{0 c}$ & $0.2546 \mathrm{~m}$ \\
\hline$x_{0 b}$ & $0.008 \mathrm{~m}$ & $V_{r}$ & $29 \cdot 10^{-3} \mathrm{~m}^{3}$ \\
\hline$A_{c i, n}$ & $24.4 \cdot 10^{-3} \mathrm{~m}^{2}$ & $A_{e b, n}$ & $6.4 \cdot 10^{-3} \mathrm{~m}^{2}$ \\
\hline$A_{r c, n}$ & $7.7 \cdot 10^{-3} \mathrm{~m}^{2}$ & $A_{b r, n}$ & $6.8 \cdot 10^{-3} \mathrm{~m}^{2}$ \\
\hline$m$ & $100 \mathrm{~kg}$ & & \\
\hline \hline
\end{tabular}

Table 1: Numerical values of parameters.

The mathematical model was tuned and verified against experimental data to show excellent compliance 
with the test cylinder. An example of simulated and experimental results for similar operating conditions are shown in Figure 3.
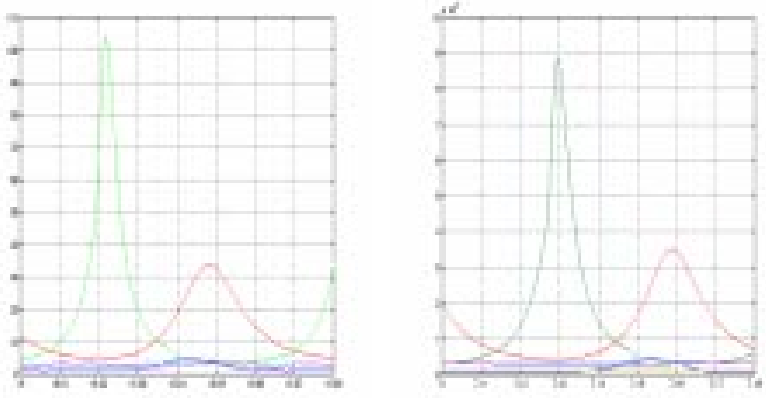

Figure 3: Model validation: Pressures $p_{b}, p_{r}, p_{a}$ and $p_{c}$ vs. time (s). Experimental results are left and simulations are right.

\section{Model Analysis}

The piston motion control subsystem is essentially required to control the TDC and $\mathrm{BDC}$ positions of the pistons, with small cycle-to-cycle variations during steady-state operation and with hard constraints during load transients. The purpose of this section is to analyse how the variables $x_{T D C}$ and $x_{B D C}$, that we want to control, can be influenced by manipulating the free variables we have at hand.

\subsection{Control variables}

The basic control variables that are available are the signals to the injector (common rail), exhaust valves and piston compressor delivery valves (both hydraulically actuated poppet valves), and in/out valves at the air cushion (electro-hydraulic cartridge valves). These are all on/off type of valves that can be commanded open or closed. These control variables offer considerable degrees of freedom, but there are also certain constraints on their use. The most useful control variables are the air cushion mass $m_{a}$ and the amount of injected diesel per cycle $m_{d i}$. Notice that the reason why $m_{d i}$ is a free variable is that the load of the engine is controlled by a supervisory controller actuating on the turbomachinery or commanding setpoints to the motion control system to achieve the desired exhaust manifold pressure when accelerating or retarding the engine. The variables $m_{d i}$ and $m_{a}$ are convenient to work with since they are more or less invariant over the cycle, in contrast to most other variables in the engine that are periodic. Notice that $m_{d i}$ can be specified directly to the diesel injection system through a mapping that maps diesel pressure and desired volume of injected diesel to electronic pulses. The air cushion mass $m_{a}$ is controllable through valves, and is the setpoint to a low level control system which includes an estimator for the air cushion mass, see Figure 4.

\subsection{Energy considerations}

Here we establish how the steady-state values of $x_{T D C}$ and $x_{B D C}$ can be influenced by $m_{d i}$ and $m_{a}$. In order to relate $m_{d i}$ and $m_{a}$ to $x_{B D C}$ we consider the expansion stroke, i.e. piston motion from TDC to BDC. The additional work on the piston needed to change the stroke length by $\Delta x_{B D C}$ near the BDC is approximately

$$
\Delta W=\left(p_{a}^{B D C} A_{a}+\left(p_{c}^{B D C}-p_{0}\right) A_{c}-p_{b}^{B D C} A_{b}\right) \Delta x_{B D C}
$$

when assuming $\triangle x_{B D C}$ is small. Furthermore, one may approximate $p_{c}^{B D C} \approx p_{b}^{B D C} \approx p_{r}$ if the pressure loss over the valves are negliable. This additional work may be produced by additional fuel input $\Delta m_{d i}$, so

$$
\begin{array}{r}
\Delta m_{d i} h_{d i}=\Delta x_{B D C} \cdot \\
\left(m_{a} A_{a} R \bar{T}_{a} \frac{\bar{V}_{a}^{\kappa-1}}{\left(V_{a}^{B D C}\right)^{\kappa}}+\left(p_{r}-p_{0}\right) A_{c}-p_{r} A_{b}\right)
\end{array}
$$

where we have used the ideal gas law and isentropic compression relations

$$
p_{a}^{B D C}=m_{a} R \bar{T}_{a} \frac{\bar{V}_{a}^{\kappa-1}}{\left(V_{a}^{B D C}\right)^{\kappa}}
$$

where $\bar{T}_{a}$ and $\bar{V}_{a}$ are the cycle-averaged volume and temperature of the air cushion. Hence, in order to change the position of the BDC by $\triangle x_{B D C}$ from one cycle to the next while keeping $m_{a}$ constant, one must add $\Delta m_{d i}$ defined by (16) to the mass of injected fuel.

The differential influence of air cushion mass $m_{a}$ and amount of injected fuel $m_{d i}$ on $x_{T D C}$ can be found by considering the compression stroke, i.e. the piston motion from BDC to TDC. The additional work on the piston needed to change the stroke length by a small amount $\triangle x_{T D C}$ near the TDC is approximately

$$
\Delta W=\left(p_{b}^{T D C} A_{b}-\left(p_{c}^{T D C}-p_{0}\right) A_{c}-p_{a}^{T D C} A_{a}\right) \Delta x_{T D C}
$$

Again, one may approximate $p_{c}^{T D C} \approx p_{i}$ and $p_{b}^{T D C} \approx$ $p_{r} r_{d i} \alpha_{d i}$, where $r_{d i}$ is the combustion chamber compression ratio, and $\alpha_{d i}>1$ is a factor that accounts for the peak pressure increase due to combustion. At idle $\alpha_{d i} \approx 2$ while at full load $\alpha_{d i}$ is only slighly larger than 1 (becuase of the long injection period at full load only a small fraction of the diesel will be combusted when the peak pressure is reached). The combustion chamber compression ratio depends on the compression ratio achieved in the piston compressor such that the total compression ratio (piston compressor plus combustion chamber) is constant $r_{t}$ :

$$
r_{d i}=r_{t} \frac{p_{i}}{p_{r}}
$$

where $r_{t}$ is a constant. Furthermore, using the ideal gas law and isentropic compression relations we get

$$
p_{a}^{T D C}=m_{a} R \bar{T}_{a} \frac{\bar{V}_{a}^{\kappa-1}}{\left(V_{a}^{T D C}\right)^{\kappa}}
$$

The change in work $\Delta W$ may be caused by a change in the internal energy of the air cushion $\Delta U_{a}=c_{v} T^{\prime} \Delta m_{a}$ by changing the amount of air by $\Delta m_{a}$

$$
\begin{aligned}
& c_{v} T^{\prime} \Delta m_{a}=\Delta x_{T D C} . \\
& \left(p_{i} r_{t} \alpha_{d i} A_{b}-\left(p_{i}-p_{0}\right) A_{c}-m_{a} A_{a} R \bar{T}_{a} \frac{\bar{V}_{a}^{\kappa-1}}{\left(V_{a}^{T D C}\right)^{\kappa}}\right)
\end{aligned}
$$

where $T^{\prime}=T_{a}$ when $\Delta m_{a}<0$ and $T^{\prime}=T_{p}$ when $\Delta m_{a}>0$. 


\subsection{Control Structure}

Simulations show that the couplings are not too strong since $m_{d i}$ influences mainly $x_{B D C}$ and $m_{a}$ influences mainly $x_{T D C}$. This can also be seen from the energy considerations in Section 4.2, cf. (16) and (20). One may therefore expect that two SISO controllers may lead to adequate performance, one controller that commands $m_{d i}$ on the basis of deviations in $x_{B D C}$ and one that commands $m_{a}$ on the basis of deviations in $x_{T D C}$. This leads to the motion control structure suggested in Figure 4.

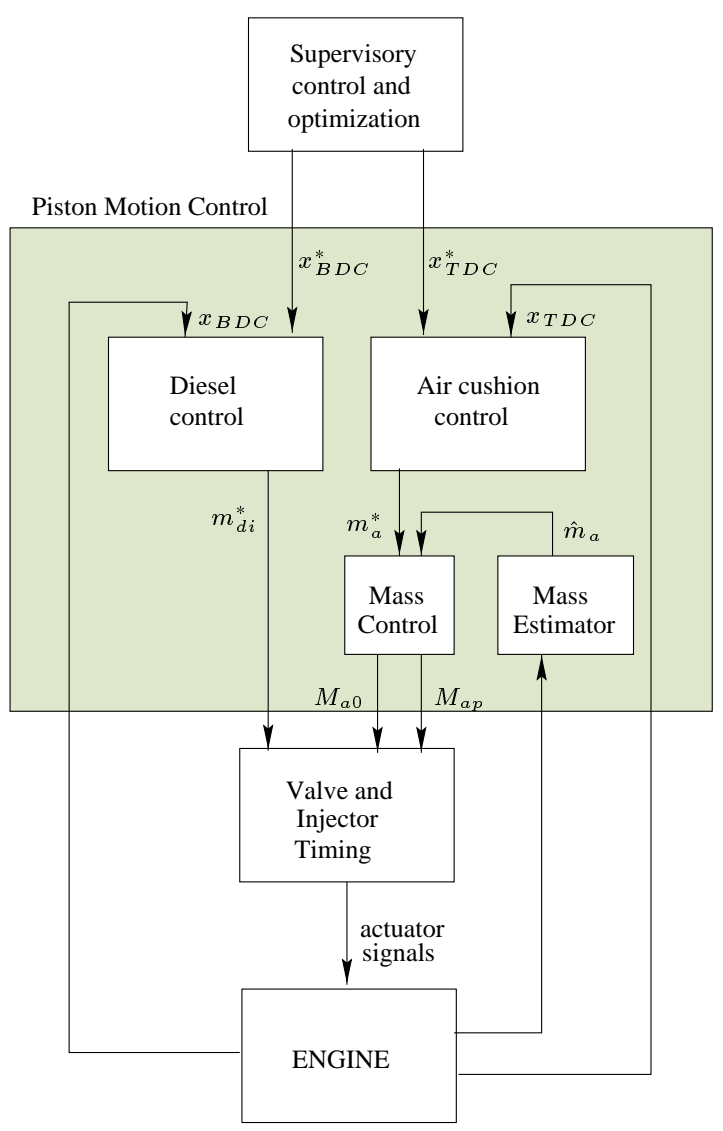

Figure 4: Motion control system structure.

Integral action is required in both control loops because it is essential that the steady-state error is small, and there may be a significant offset in the diesel injection system such that the actual $m_{d i}$ differs from the commanded $m_{d i}^{*}$. Furthermore, there may be a large offset also in the air cushion mass estimator due to a poor guess of the mean temperature such that the actual $m_{a}$ differs from its estimate $\hat{m}_{a}$. Because the response from $m_{d i}$ to $x_{B D C}$ is open loop unstable, derivative action is useful to get high performance and robustness.

The results from model analysis suggest the relationships (16) and (20) that can be rewritten in the form

$$
\begin{aligned}
\Delta m_{d i} & =\left(k_{0}+k_{1} m_{a}+k_{2} p_{r}\right) \Delta x_{B D C} \\
\Delta m_{a} & =\left(g_{0}+g_{1} m_{a}+g_{2} p_{r}+g_{3} p_{i}\right) \Delta x_{T D C}
\end{aligned}
$$

where $k_{0}, k_{1}, k_{2}, g_{0}, g_{1}, g_{2}$ and $g_{3}$ are constants. Obviously, this suggests some controller nonlinearities. The variables $m_{a}, p_{i}$ and $p_{r}$ are roughly invariant over each cycle and depends mainly on the load of the engine. At idle, $p_{i}=1$ bar and it increases gradually to $p_{i}=3.25$ bar at full load. Morover, $p_{r}=2$ bar at idle and it increases to $p_{r}=22$ bar at full load. Moreover, $m_{a}=16$ $\mathrm{g}$ at idle and it increases to about $m_{a}=80 \mathrm{~g}$ at full load. Figure 5 illustrates theoretical dependencies of $x_{T D C}^{*}, x_{B D C}^{*}, m_{a}^{*}, m_{d i}^{*}, p_{r}$ and $p_{i}$ as a function of load. These are computed in order to achieve an exhaust gas temperature that is independent of the load. Thus, one may rewrite (21) and (22) as

$$
\begin{aligned}
\Delta m_{d i} & =k(L) \Delta x_{B D C} \\
\Delta m_{a} & =g(L) \Delta x_{T D C}
\end{aligned}
$$

where $L$ is load varying between approximately $5 \%$ at idle to $100 \%$. The gains are illustrated in Figure 6 . It should be noted that even though the individual terms of (22) are highly nonlinear (for example $g_{2} p_{r}$ will change with a factor of more than 10 from idle to full load) it is clear that (24) are much less nonlinear as a function of $L$ because the individual terms will dominate at different loads.
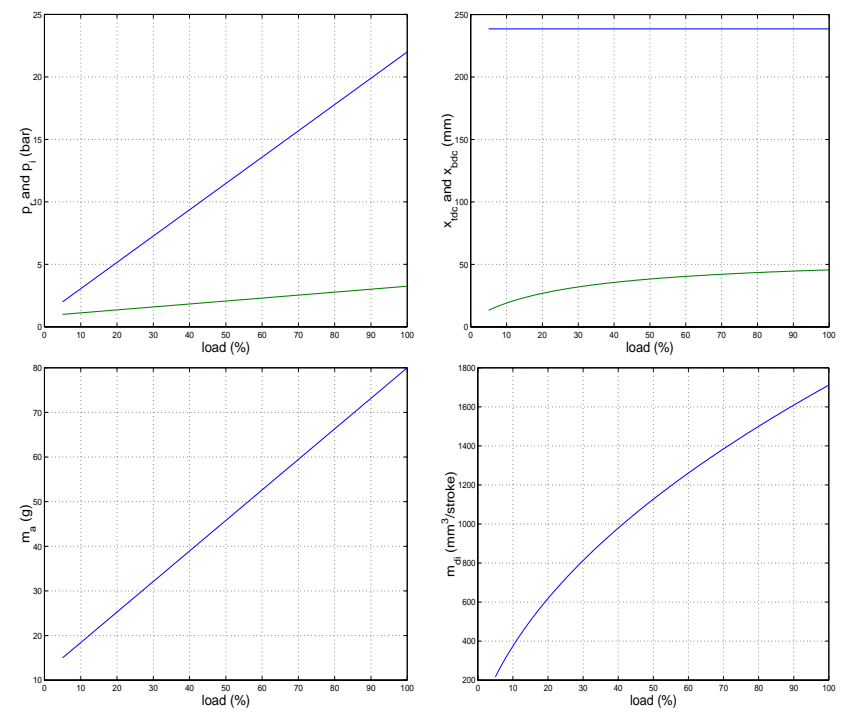

Figure 5: Main engine parameters as a function of load (theoretical calculations).
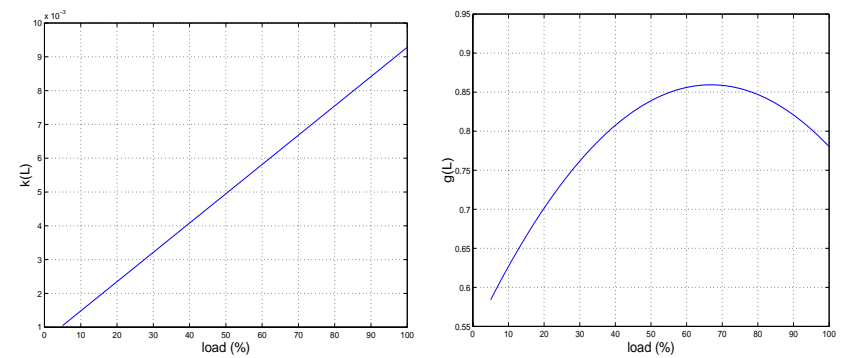

Figure 6: Nonlinear gain as a function of load.

The main contributions to the dynamics is the volume of the interstage receiver chamber. A rough estimate of the dominant time-constant might be found by computing the ratio of the volume $V_{r}$ to the volumetric flow rate $q_{r}=\bar{M}_{r} / \rho_{r}$, where $\bar{M}_{r}$ is the mass flow through the interstage receiver averaged over a cycle. Notice that $V_{r}$ is constant, while $q_{r}$ may show some variation 
between idle and full load. However, this variation is neglectable since the air-to-fuel ratio does not vary too much (it is somewhat higher at idle than at full load). Hence, $\tau=V_{r} / q_{r}$ can be considered to be operating point independent. In fact, experimental results shows that adequate performance can be achieved with linear controllers (PI control of the air cushion and PID control of the diesel injection) over the full operating range, even though a nonlinear diesel controller where the gain is scheduled on the load of the engine consistent with (23) might give consistently better performance over the full operating range.

\section{Experimental Results}

Results of transient running of the $1 \mathrm{MW}$ test cylinder are shown in Figure 7, see [3] for details on the experimental setup. Initially, the piston is at rest at the PBS position and the pressure in the engine is atmospheric. The engine starts at $t \approx 2.0 \mathrm{~s}$ by injecting the exact amount of air $m_{a}$ into the air cushion to generate the pressure required to make the piston move to the desired TDC postion. At the TDC exactly the required amount of diesel $m_{d i}$ is injected to take the piston to the desired BDC position. At this point, the piston motion controller takes over, and command $m_{a}$ and $m_{d i}$ for each cycle. The engine load is increased from idle to about $40 \%$ during this time interval, by manually choking the exhaust gas flow. We observe from the curves that the piston motion control system operates satisfactory with variability of the dead center position of about $1 \mathrm{~mm}$ after the initial transient. Somewhat larger variability must be accepted during startup.

\section{Conclusion}

A detailed dynamic model of a free-piston diesel engine is derived based on thermodynamics relations and the equations of motion, leading to a set of ordinary differential equations. The dynamic analysis reveal that the piston motion can be stabilized and the top and bottom dead center positions can be controlled by manipulating the air cushion mass and the amount of injected diesel per cycle. A simple control structure for this purpose is suggested and verified experimentally on a full scale test cylinder.

\section{References}

[1] A. F. Moiroux, "Free-piston engine possibilities", in ASME Oil and Gas Power Conference, 1958, pp. 58OGP-7.

[2] S. Tikkanen and M. Vilenius, "Hydraulic free piston engine - challenge for control", in Proc. European Control Conference, Karlsruhe, 1999.

[3] T. A. Johansen, O. Egeland, E. A. Johannessen, and R. Kvamsdal, "Free-piston diesel engine timing and control - towards electronic cam- and crankshaft", IEEE Trans. Control Systems Technology, vol. 9, 2001. [4] M. J. Førde, T. A. Johansen, R. Kvamsdal, and O. Egeland, "A method for controlling the stroke of a diesel free-piston gas generator", Patent WO9728362, 1997.

[5] J. B. Heywood, Internal Combustion Engine Fundamentals, McGraw-Hill, 1988.

[6] G. Borman and K. Ragland, Combustion Engineering, McGraw-Hill, 1998.
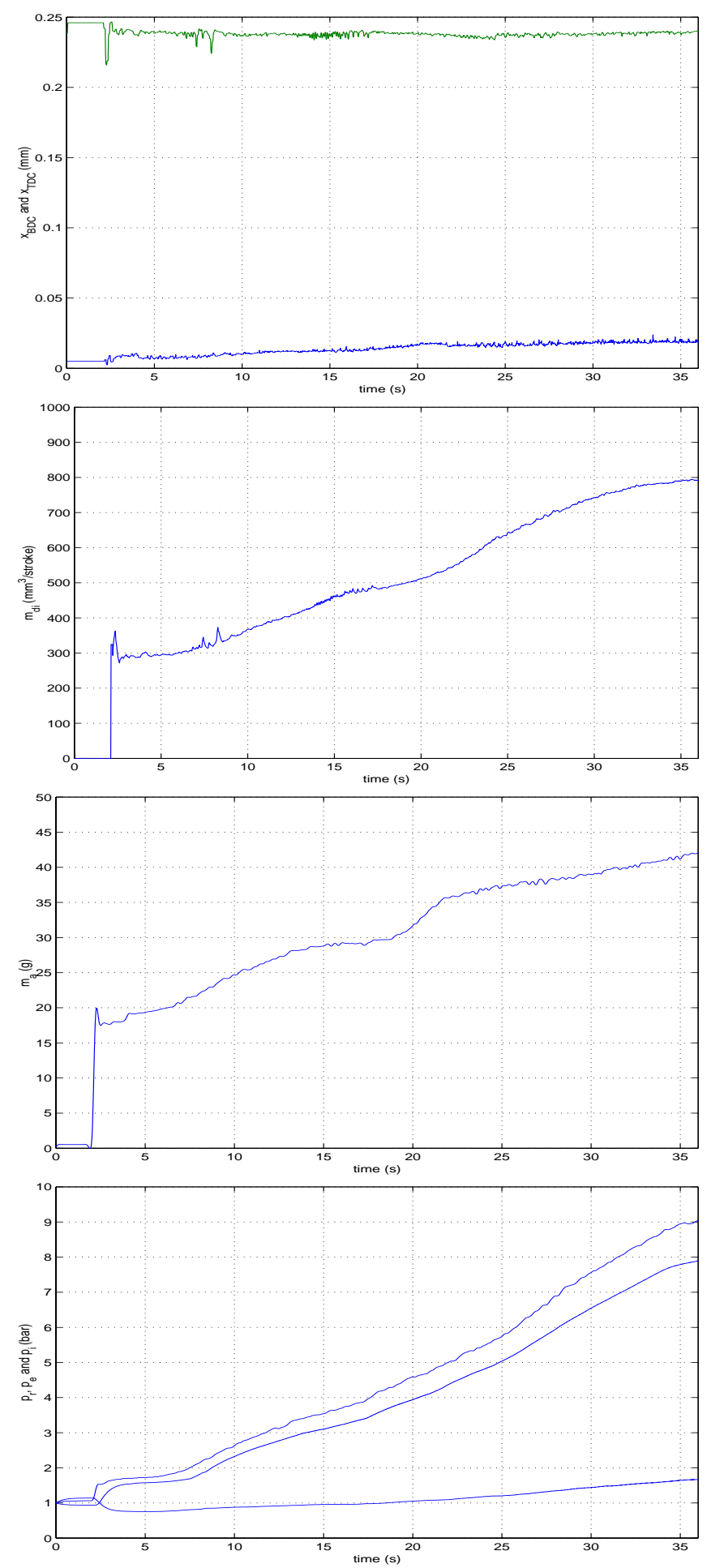

Figure 7: Piston motion ( $x_{B D C}$ and $x_{T D C}$ positions $)$ and control input $m_{d i}$ and $m_{a}$ when the load of the engine is increased from idle to about $40 \%$ using a throttle in the exhaust pipe. Curves for the exhaust manifold pressure $p_{e}$, scavenging pressure $p_{r}$ and intake manifold pressure $p_{i}$ are also shown. 